\title{
Parathyroid Hormone-Related Protein Is Induced during Lethal Endotoxemia and Contributes to Endotoxin-Induced Mortality in Rodents
}

\author{
Janet L. Funk, Arthur H. Moser, Gordon J. Strewler, \\ Kenneth R. Feingold, and Carl Grünfeld \\ Department of Medicine, University of California, San Francisco, San \\ Francisco, California, U.S.A. \\ Metabolism and Endocrinology Sections, Veterans Administration \\ Medical Center, San Francisco, California, U.S.A.
}

\begin{abstract}
Background: Parathyroid hormone-related protein (PTHrP) is a ubiquitous and highly conserved vasoactive peptide whose role and regulation in normal physiology remain an enigma. Recently, we demonstrated that lowdose endotoxin (LPS) induces intrasplenic, but not systemic, levels of PTHrP; and that tumor necrosis factor, a pro-inflammatory cytokine, is the major mediator of this effect. We have therefore hypothesized that, with higher, lethal doses of endotoxin, PTHrP could be induced in multiple tissues to such a degree that it could contribute to the lethality of septic shock.

Materials and Methods: Northern blot analysis was used to measure PTHrP mRNA levels in vital organs of rats after administration of a near lethal dose $(5 \mathrm{mg} / 250$ g) of LPS (or vehicle alone). Plasma levels of PTHrP were also measured by immunoradiometric assay. The ability
\end{abstract}

of the immunoglobulin fraction of two different PTHrP(1-34) antisera to protect from LPS-induced lethality was also studied in mice using survival analysis. Results: In response to a near-lethal dose of endotoxin, PTHrP mRNA levels increased acutely in every vital organ examined (spleen, lung, heart, kidney, and liver). Circulating levels of PTHrP also increased, peaking $2 \mathrm{hr}$ after administration of highdose endotoxin. Passive immunization of mice with anti-PTHrP(1-34) antibody $6 \mathrm{hr}$ prior to administration of a lethal dose of LPS protected mice from endotoxin-induced death $(p<0.00005)$.

Conclusions: These results suggest that PTHrP belongs to the cascade of pro-inflammatory cytokines induced during lethal endotoxemia that is responsible for the toxic effects of LPS.

\section{INTRODUCTION}

With the identification of parathyroid hormonerelated protein (PTHrP) in the late 1980s, the search for the parathyroid hormone (PTH)-like mediator of hypercalcemia of malignancy ended and a new search began to determine the role and regulation of this ubiquitous and highly conserved peptide in normal physiology. While sustained elevations in circulating levels of tumor-derived PTHrP cause hypercalcemia of malignancy via interaction with PTH/PTHrP recep-

Address correspondence and reprint requests to: Janet L. Funk, Arizona Health Science Center, PO 245021, 1501 North Campbell, Tucson, AZ 85724, U.S.A. tors in classic PTH target tissues (kidney and bone), circulating PTHrP is barely detectable in normal individuals $(1,2)$. However, both PTHrP and the recently cloned PTH/PTHrP receptor are widely expressed in normal tissues, thus suggesting that PTHrP normally acts at its site of production in a paracrine or autocrine fashion more akin to cytokines than to classic hormones, such as PTH (1-3).

Recently, we reported that PTHrP gene expression is stimulated in the spleen during the host response to sublethal doses of endotoxin (lipopolysaccharide, LPS) (4). Tumor necrosis factor (TNF), an important pro-inflammatory cytokine, mediates LPS induction of splenic PTHrP 
(4). Other cytokines also modulate splenic PTHrP expression $(4,5)$. These studies provide evidence that complex cytokine networks may regulate local PTHrP expression and suggest that PTHrP may play a role in the host response to inflammation and infection.

The injection of a lethal dose of endotoxin (1000-fold higher than doses used in our earlier experiments) can be used to model sepsis, a systemic inflammatory response to overwhelming infection by gram-negative or other bacteria $(6-8)$. Although the precise cause of death from sepsis is unknown, hypotension is a hallmark of lethal endotoxemia (6-8). Alterations in vascular hemodynamics are also thought to contribute to the multisystem organ failure that accompanies septic shock $(6-8)$. The important role of cytokines in mediating the lethal effects of septic shock is well established. For example, the decrease in systemic vascular resistance (due to vasodilation) that occurs in septic shock also occurs in response to TNF, a cytokine that is transiently induced after endotoxin administration (6-9), and passive immunization of mice with antibody directed against TNF protects from the lethal effects of endotoxin (10).

PTHrP is also a known vasodilator that can be produced by and cause relaxation of smooth muscle cells (11-14). In addition, our earlier low-dose endotoxin studies showed that smooth muscle cells were the source of LPS-induced splenic PTHrP (15). Because a decrease in vascular resistance is seen both systemically and locally within organs during lethal endotoxemia, we hypothesized that acute elevations in local and/or systemic levels of PTHrP during sepsis could contribute to the lethal effects of endotoxin. To test this hypothesis, we investigated PTHrP gene expression in rats treated with a near-lethal dose of endotoxin. In addition, the ability of PTHrP neutralizing antibody to protect from LPS-induced death was also studied.

\section{MATERIALS AND METHODS}

\section{Materials}

Escherichia coli, strain O55:B5 endotoxin was obtained from Difco Laboratories (Detroit, MI, U.S.A.). The murine PTHrP complementary DNA (cDNA) probe, which includes the region encoding amino acids $1-114$ of the mature peptide, was kindly provided by Dr. Arthur E. Broadus (Yale University School of Medicine, New Haven, CT, U.S.A.).
Antibodies were generated against synthetic human PTHrP(1-34) (Bachem, Torrance, CA, U.S.A.) by immunization of rabbits or goats using standard techniques at Berkeley Antibody Company (Berkeley, CA, U.S.A.). The immunoglobulin fraction of immune (PTHrP antibody) or naive sera (control antibody) was partially purified by ammonium sulfate precipitation ( $33 \%$ saturation) using previously described techniques to avoid the introduction of endotoxin contamination (16). Endotoxin content of PTHrP and control antibody solutions ( $\leq 165 \mathrm{pg} /$ mouse), determined by Limulus assay as previously described (5), was below the level required to alter sensitivity to subsequent (6 h) LPS challenge in mice $(17,18)$.

\section{Animal Procedures}

Male Sprague-Dawley rats, 200-220 g, were purchased from Simonsen (Gilroy, CA, U.S.A.). Rats were injected via tail vein with $5 \mathrm{mg} / 250 \mathrm{~g}$ weight LPS diluted in apyrogenic $0.9 \%$ saline (Kendall McGraw Laboratories, Irvine, CA, U.S.A.) or with vehicle alone. Immediately after receiving this dose of endotoxin, the animals appeared sick and developed diarrhea. However, no deaths occurred prior to sacrifice $(t=0-14$ $\mathrm{hr}$ ) for harvesting of vital organs and/or plasma $\left(\mathrm{LD}_{50}=10 \mathrm{mg} / 250 \mathrm{~g}\right.$ at $t=14 \mathrm{hr}$ for the lot of LPS used).

Male 4- to 5-week-old C57BL/6 mice, purchased from The Jackson Laboratory (Bar Harbor, ME, U.S.A.), were injected i.p. with PTHrP or control antibody $6 \mathrm{hr}$ prior to i.p. injection of LPS $(650-800 \mu \mathrm{g} / \mathrm{mouse}$, corresponding to an $\mathrm{LD}_{\geq 80}$ by $48 \mathrm{hr}$ ). The volume of goat or rabbit antibody injected from different bleeds was adjusted so that an equivalent titer of PTHrP antibody, as determined by ELISA, was administered in all experiments reported here (corresponding to $100 \mu \mathrm{l}$ of titer $1: 16,000)$. No lethality was observed in control animals administered antiPTHrP or control antibodies (goat or rabbit) $6 \mathrm{hr}$ prior to i.p. injection of vehicle alone.

\section{Northern Blot Analysis}

Polyadenylated mRNA was isolated from rat spleen, lungs, heart, kidneys, or liver for Northern analysis utilizing $\left[{ }^{32} \mathrm{P}\right] \mathrm{CDNA}$ probes as previously described (4). Because no "housekeeping" gene could be found that remained unchanged in all organs in response to near-lethal doses of LPS, normalized PTHrP mRNA levels are not reported. However, in organs where actin and/or 
cyclophilin mRNA levels were unchanged (heart, kidney, and liver), normalization of PTHrP mRNA levels did not alter results (data not shown). Northern blots for all organs were also probed for other cytokines (TNF, interleukin-1, and interleukin-6) to verify that variable time courses of induction for these genes also occurred, consistent with prior reports (data not shown).

\section{Immunoradiometric Assay of PTHrP}

Blood drawn from the inferior vena cava of anesthetized rats was immediately mixed with heparin and protease inhibitors (leupeptin $2.6 \mu \mathrm{g} / \mathrm{ml}$ blood; aprotinin $43.2 \mu \mathrm{g} / \mathrm{ml}$ blood) and placed on ice prior to centrifugation for separation of plasma. PTHrP immunoreactivity was measured in plasma samples with a two-site PTHrP immunoradiometric assay (sensitivity, $0.3 \mathrm{pM}$ ) that utilizes antibodies directed against synthetic human PTHrP(1-40) and PTHrP(60-72) (Nichols Institute, San Juan Capistrano, CA, U.S.A.).

\section{Statistical Analysis}

Northern analysis and immunoradiometric assay values are presented as mean \pm SEM with statistical significance determined by ANOVA and $p$ values computed by two-tailed Student's $t$ test using InStat 2.01 (GraphPad, Software, San Diego, CA, U.S.A.). Kaplan Meier survival curves were generated and analyzed by either log-rank or Peto \& Peto Wilcoxon test using Statistica 4.1 (StatSoft, Tulsa, OK, U.S.A.). For a given time point, statistical significance of lethality rates was determined by Fisher's exact test (InStat 2.01).

\section{RESULTS}

\section{Effect of Near-Lethal Dose of LPS on PTHrP Gene Expression in Vital Organs of the Rat}

Since PTHrP is known to be present constitutively in many of the vital organs that fail during septic shock, we hypothesized that the local induction of PTHrP in these organs could be associated with the multisystem failure that occurs during lethal endotoxemia. To test this hypothesis, PTHrP gene expression in the spleen, lung, heart, kidney, and liver of rats treated with a near-lethal $(5 \mathrm{mg} / 250 \mathrm{~g}$ ) dose of endotoxin was determined by Northern analysis. A dramatic increase in PTHrP mRNA levels occurred acutely in all vital organs examined in response to LPS. Maximal induction of PTHrP mRNA occurred earliest in spleen and lung (45-90 min) (Fig. 1A), followed by heart (1.5 hr) (Fig. 1B) and kidney $(2 \mathrm{hr})$ (Fig. 1C). Induction of hepatic PTHrP mRNA was even more delayed, increasing throughout the $3.5 \mathrm{hr}$ following LPS treatment (Fig. 1C) and returning to baseline by $6 \mathrm{hr}$ (data not shown).

\section{Effect of Near-Lethal Dose of LPS on Plasma Levels of PTHrP in Rats}

In our earlier low-dose studies, endotoxin increased intrasplenic, but not circulating levels, of PTHrP peptide (4). However, if the striking induction of PTHrP mRNA levels found in all vital organs in response to near-lethal doses of endotoxin was accompanied by comparable increases in PTHrP secretion, we hypothesized that circulating levels of PTHrP could also be elevated during lethal endotoxemia. As predicted, plasma levels of PTHrP were indeed increased in rats administered near-lethal doses of endotoxin (Fig. 2). Circulating levels of $\mathrm{N}$-terminal-containing PTHrP peptides were increased at the earliest time point studied $(45 \mathrm{~min}$ ) and peaked at $2 \mathrm{hr}$, increasing to $1.36 \mathrm{pM}$. By $3.5 \mathrm{hr}$, when splenic, pulmonary, cardiac, and renal (but not hepatic) PTHrP mRNA levels were near baseline, circulating levels of immunoreactive PTHrP returned towards baseline. At later times (6-14 hr), plasma levels of PTHrP were actually lower than constitutive circulating levels of PTHrP.

\section{Effect of PTHrP Antibody on LPS-Induced Lethality in Mice}

To test our hypothesis that PTHrP could mediate some of the toxic effects of LPS, mice were passively immunized, prior to the administration of a lethal dose of endotoxin, with antibody generated against PTHrP (1-34), a peptide fragment that is active at the PTH/PTHrP receptor. Administration of goat PTHrP antibody $6 \mathrm{hr}$ prior to the injection of LPS significantly protected mice from death compared with the mortality rate seen in mice treated with control antibody $(p<0.03$, Kaplan Meier survival analysis) (Fig. 3A). The protective effect of PTHrP antibody was also confirmed by studies utilizing antiserum raised in rabbits against PTHrP(1-34) $(p<0.004)$ (Fig. 3B).

While comparison of survival curves showed that PTHrP antibody provided a significant protective effect from LPS-induced death over the 3- 

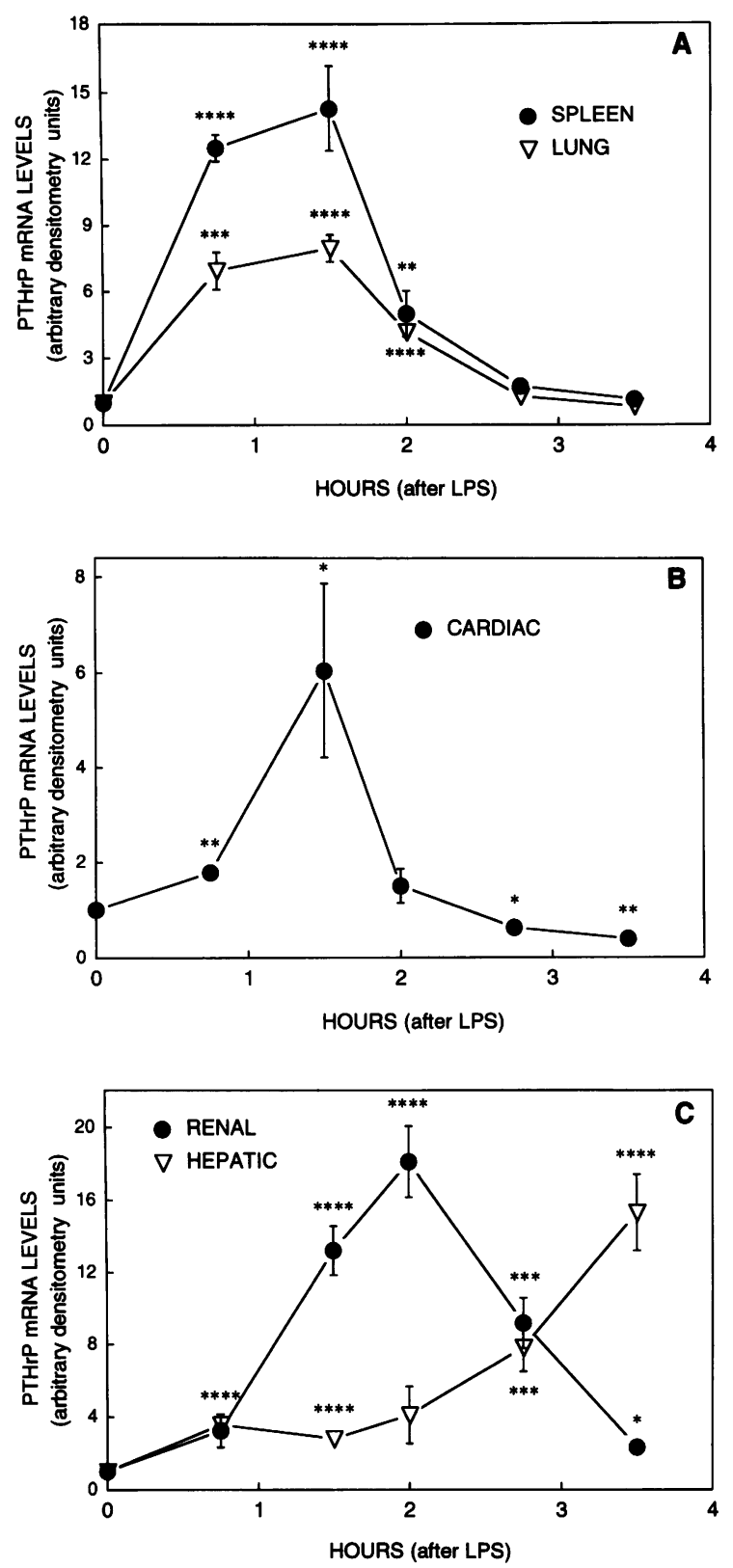

FIG. 1. Effect of a near-lethal dose of endotoxin on PTHrP mRNA levels in vital organs

Male Sprague Dawley rats were injected via tail vein with $5 \mathrm{mg} / 250 \mathrm{~g}$ body weight Escherichia coli strain 055:B5 LPS. At indicated times, spleen (A; circle), lungs (A; triangle), heart $(B)$, kidneys (C; circle), and liver $(\mathrm{C}$; triangle) were removed for Northern analysis of polyadenylated mRNA using a murine PTHrP cDNA probe (4). PTHrP mRNA is reported as mean $\pm \operatorname{SEM}(n=4 /$ time $)$. Differences are statistical significant by ANOVA; $p$ values determined by twotailed Student's $t$ test. ${ }^{*} p<0.05$ (versus $t=0$ ); ${ }^{* *} p<0.01 ; * * * p<0.005 ; * * * * p<0.001$.

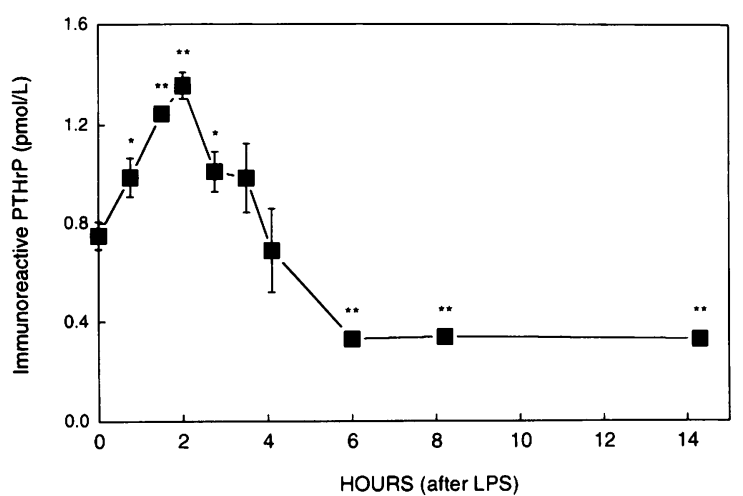

FIG. 2. Effect of near-lethal dose of endotoxin on plasma levels of PTHrP

At times indicated, blood was drawn from the inferior vena cava of anesthetized rats treated with LPS as in Fig. 1. Plasma PTHrP was measured by immunoradiometric assay utilizing antibodies directed against PTHrP (1-40) and PTHrP (60-72) (Nichols). Results (mean \pm SEM; $n=3-7 /$ group) are representative of three experiments. Differences are statistical significant by ANOVA; $p$ values determined by twotailed Student's $t$ test. ${ }^{*} p<0.05$ (versus $t=0$ ); ${ }^{* *} p<0.001$.

to 4-day course of experiments (Fig. $3 \mathrm{~A}$ and $\mathrm{B}$ ) (Kaplan Meier survival analysis), at the end of the observation periods the relative survival rates were no different (Fisher exact test). Compilation of data from multiple experiments $(n=90$ mice) examining the ability of rabbit PTHrP antibody to protect from LPS-induced death confirms this conclusion. While overall survival was improved by pretreatment with rabbit PTHrP antibody ( $p<0.00005$, Kaplan Meier survival analysis), comparison of lethality rates at 12 -hr intervals following LPS only showed a significant protective effect during the first $48 \mathrm{hr}$ (Fig. 4). Therefore, pretreatment with PTHrP antibody delayed, but ultimately did not prevent, endotoxin-induced death.

\section{DISCUSSION}

While PTHrP has been identified and defined by its relation to PTH, a hormone that follows the normal endocrine paradigm of localized production and distant action, newer evidence suggests that PTHrP may in fact act more like a cytokine than a classic hormone, being widely expressed in normal tissues by a variety of cell types, where it is thought to have local autocrine or paracrine effects $(1,2)$. Therefore, alterations in bone and 

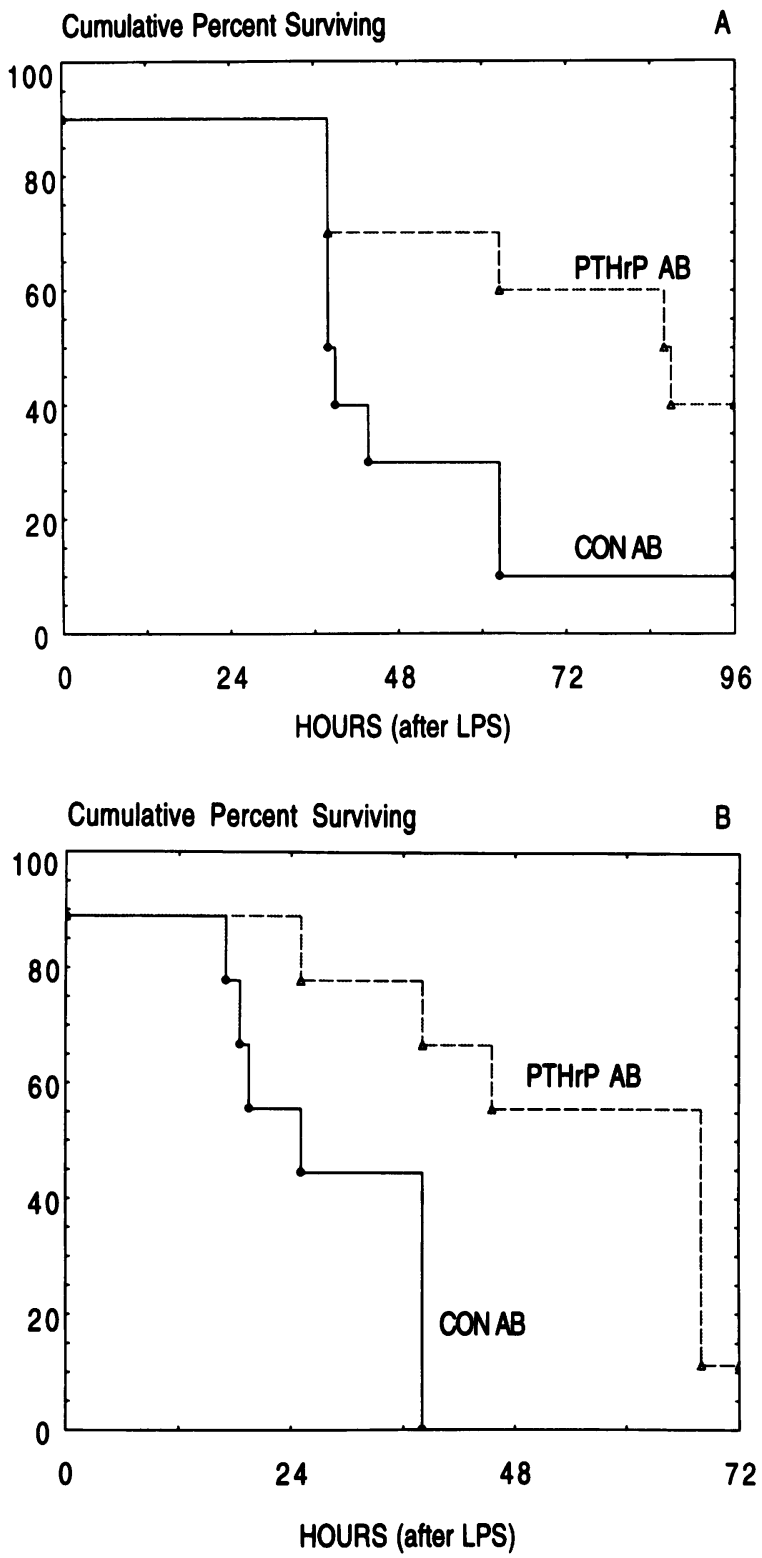

FIG. 3. Effect of goat (A) or rabbit (B) PTHrP (1-34) antibody on LPS-induced lethality

(A) Male C57BL/6 5- to 6-week-old mice ( $n=$ 9/group) were injected i.p. with $200 \mu$ l goat PTHrP antibody (titer 1:8,000 by ELISA) (triangle) or goat control antibody (circle) $6 \mathrm{hr}$ prior to LPS $(700 \mu \mathrm{g}$ i.p.). Statistical analysis of Kaplan Meier survival curves using either a log-rank (all time points equally weighted) or Peto \& Peto Wilcoxon test (earlier time points more heavily weighted) showed that pretreatment with PTHrP antibody was protective ( $p<0.030$ and $p<0.031$, respectively). (B) Mice $(n=8$ /group) were injected with $100 \mu$ l rabbit PTHrP antibody (titer l:16,000) (triangle) or rabbit control antibody (circle) $6 \mathrm{hr}$ prior to LPS (700 $\mu \mathrm{g}$ i.p.). Pretreatment with PTHrP antibody was protective $(p<$ 0.004 log-rank; $p<0.003$ Peto \& Peto Wilcoxon).

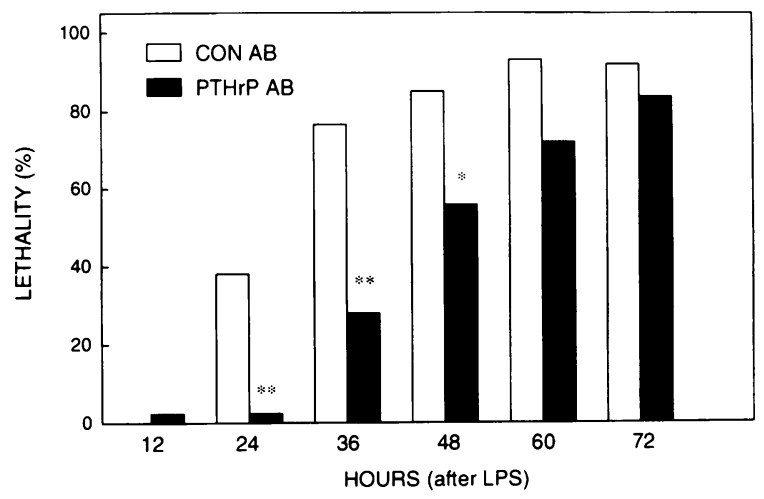

FIG. 4. Effect of rabbit PTHrP(1-34) antibody on LPS-induced lethality

Lethality data from five separate experiments, performed as in Fig. 3, were combined for analysis. In each experiment, mice from control antibody and PTHrP antibody treatment groups were injected with an equal amount of LPS (650-800 $\mu \mathrm{g}$ LPS, corresponding to $\mathrm{LD}_{\geq 80}$ at $t=48 \mathrm{hr}$ in control antibodytreated mice). Statistical analysis of Kaplan Meier survival curves (not shown here) for PTHrP $(n=43)$ and control $(n=47)$ antibody-treated groups showed that PTHrP antibody was protective $(p<$ 0.00005 log-rank; $p<0.000001$ Peto and Peto Wilcoxon). Lethality data for control antibody-treated (open bar) and PTHrP antibody-treated (filled bar) mice was also compared at 12 -hr intervals after LPS (Fisher's exact test). ${ }^{*} p<0.003 ;{ }^{* *} p<0.0001$.

calcium metabolism may be but one of many important biological functions subserved by this ubiquitous peptide.

In a series of earlier reports, we have shown that sublethal doses of LPS induce PTHrP gene expression locally in the spleen and that TNF, an important proinflammatory cytokine, is the major mediator of this effect while other pro- and antiinflammatory cytokines also modulate PTHrP splenic expression $(4,5)$. These studies add a new dimension to the current view of PTHrP as a cytokine, suggesting that PTHrP may in fact be one additional member of the cascade of cytokines induced during the host response to infection and inflammation.

We previously found that splenic stromal cells, including smooth muscle cells, are the source of splenic PTHrP; and that mRNA for both PTHrP, a vasodilatory peptide (11-14), and the PTH/PTHrP receptor colocalize within the splenic stroma (15). These data suggest that PTHrP may be mediating smooth muscle relaxation at its site of production in the spleen during the host response to infection. Given the important role 
that altered hemodynamics play in septic shock, a state of profound hypotension and multiorgan failure resulting from overwhelming bacterial infection, we have now administered lethal or near-lethal doses of endotoxin to rodents to determine whether PTHrP is an important mediator of the host response to lethal endotoxemia.

During the host response to near-lethal endotoxemia, PTHrP gene expression was induced in all vital organs examined (spleen, lung, heart, kidney, and liver). It is of interest to note that the order of LPS induction of PTHrP in these organs mirrored the usual order of organ failure in sepsis, with lung preceding kidney and liver (8). In addition, although PTHrP gene expression has previously been reported in fetal rat livers, the LPS induction of hepatic PTHrP shown here is, to our knowledge, the first demonstration of PTHrP gene expression in adult rat liver (19-21). This finding suggests that PTHrP may indeed have important hepatic effects in the adult rat during the host response to infection and inflammation.

Accompanying the induction of PTHrP gene expression in multiple vital organs, circulating levels of PTHrP were also increased at an early time point during lethal endotoxemia. Similar acute, but transient, increases in circulating peptide levels have also been reported for other important cytokine mediators of the host response to endotoxemia, such a TNF (9).

Having thus documented that PTHrP induction occurs both locally and systemically during endotoxin-induced sepsis, antibody protection studies confirmed our hypothesis that PTHrP contributes to the toxic effects of endotoxin; pretreatment with goat or rabbit antibodies directed against PTHrP (1-34) prolonged survival in mice injected with a lethal dose of endotoxin. Antibody blockade experiments, similar to those presented here, have previously shown that several other cytokines, such as TNF, interleukin-1, macrophage inhibitory factor and interferon- $\gamma$, are also mediators of the lethal effects of endotoxin $(10,22-26)$. The fact that passive immunization against PTHrP delayed, but did not ultimately prevent, LPS-induced death is consistent with the hypothesis that PTHrP is one member of a larger cascade of pro-inflammatory cytokines that is unleashed during the host response to infection. Indeed, the degree of protection from LPS lethality seen here with passive immunization of mice against PTHrP is similar to that reported for passive immunization of mice against TNF, a cytokine that is a major mediator of sepsis (10).
The ability of antibodies directed against the $\mathrm{N}$-terminal portion of PTHrP, a peptide fragment that binds to and activates the PTH/PTHrP receptor, to protect against LPS-induced lethality suggests that the toxic effects of PTHrP may be mediated by the PTH/PTHrP receptor. Future studies will need to address this and other questions, thereby not only enhancing our understanding of the important physiologic effects of PTHrP, but also hopefully providing insights into potential treatments for septicemia, a condition that, despite an enormous arsenal of antimicrobials, remains the leading cause of death in intensive care units in the United States.

\section{ACKNOWLEDGMENTS}

This work was supported in part by grants from the National Institutes of Health (DK-47846 to JLF), the Research Service of the Department of Veterans Affairs (to JLF, KRF, and CG), and the University of California, San Francisco Academic Senate (to JLF).

\section{REFERENCES}

1. Stewart AF, Broadus AE. (1990) Clinical review 16. Parathyroid hormone-related proteins: Coming of age in the 1990s. J. Clin. Endocrinol. Metab. 71: 1410-1414.

2. Strewler GJ, Nissenson RA. (1990) Hypercalemia in malignancy. West. J. Med. 153: 635640.

3. Urena $\mathrm{P}$, Kong $\mathrm{XF}$, Abou-Samra $\mathrm{AB}$, et al. (1993) Parathyroid hormone/parathyroid hormone-related peptide receptor mRNAs are widely distributed in rat tissues. Endocrinology 133: 617-623.

4. Funk JL, Krul EJT, Moser AH, et al. (1993) Endotoxin increases parathyroid hormonerelated protein mRNA levels in mouse spleen: Mediation by tumor necrosis factor. J. Clin. Invest. 92: 2546-2552.

5. Funk JL, Shigenaga JK, Moser AH, et al. (1994) Cytokine regulation of parathyroid hormone-related protein mRNA levels in mouse spleen: Paradoxical effects of interferon-gamma and interleukin-4. Endocrinology 135: 351-358.

6. Bone RC. (1991) The pathogenesis of sepsis. Ann. Int. Med. 115: 457-469.

7. Parrillo JE. (1993) Pathogenetic mechanisms 
of septic shock. N. Engl. J. Med. 328: 14711477.

8. Dal Nogare AR. (1991) Southwestern Internal Medicine Conference: Septic shock. Am. J. Med. Sci. 302: 50-65.

9. Dinarello CA. (1991) The proinflammatory cytokines IL-1 and TNF and treatment of the septic shock syndrome. J. Infect. Dis. 163: 1177-1184.

10. Beutler B, Milssark IW, Cerami AC. (1985) Passive immunization against cachectin/tumor necrosis factor protects mice from lethal effect of endotoxin. Science 229: 869-871.

11. Nickols GA, Nana AD, Nickols MA, Dipette DJ, Asimakis GK. (1989) Hypotension and cardiac stimulation due to the parathyroid hormone-related protein, humoral hypercalcemia of malignancy factor. Endocrinology 125: 834-841.

12. Orloff JJ, Wu TL, Stewart AF. (1989) Parathyroid hormone-like proteins: biochemical response and receptor interactions. Endocr. Rev. 10: 476-495.

13. Rocha-Cusachs A, Dipette DJ, Nickols GA. (1991) Regional and systemic hemodynamic effects of parathyroid hormone-related protein: Preservation of cardiac function and coronary and renal flow with reduced blood pressure. J. Pharmacol. Exp. Ther. 256: 110118.

14. Thiede MA, Nickols GA. (1995) Local expression and action of parathyroid hormone-related protein in the cardiovascular system. In: Crass MF, Avioli LV (eds). Calcium-Regulating Hormones and Cardiovascular Function. CRC Press, Ann Arbor, MI, pp. 131-174.

15. Funk JL, Lausier J, Moser AH, et al. (1995) Endotoxin induces parathyroid hormone-related protein gene expression in splenic stromal and smooth muscle cells, not in splenic lymphocytes. Endocrinology 136: 3412-3421.

16. Funk JL, Feingold KR, Moser AH, Grunfeld C. (1993) Lipopolysaccharide stimulation of RAW 264.7 macrophages induces lipid accumulation and foam cell formation. Atherosclerosis 98: 67-82.

Contributed by A. Cerami on January 4, 1996.
17. Greer GG, Rietschel E. (1978) Inverse relationship between the susceptibility of lipopolysaccharide (lipid A)-pretreated mice to the hypothermic and lethal effect of lipopolysaccharide. Infect. Immun. 20: 366-374.

18. Freundenberg MA, Galanos C. (1988) Induction of tolerance to lipopolysaccharide (LPS)-D-galactosamine lethality by pretreatment with LPS is mediated by macrophages. Infect. Immun. 56: 1352-1357.

19. Thiede MA, Rodan GA. (1988) Expression of a calcium-mobilizing parathyroid hormonelike peptide in lactating mammary tissue. Science 242: 278-280.

20. Ikeda K, Weir EC, Mangin M, et al. (1988) Expression of messenger RNA encoding a parathyroid hormone-like peptide in normal human and animal tissues with abnormal expression in human parathyroid adenomas. Mol. Endocrinol. 2: 1230-1236.

21. Mangin M, Ikeda K, Broadus AE. (1990) Structure of the mouse gene encoding parathyroid hormone-related peptide. Gene 95: 195-202.

22. Tracey KJ, Fong Y, Hesse DG, et al. (1987) Anti-cachectin/TNF monoclonal antibodies prevent septic shock during lethal bacteraemia. Nature 330: 662-664.

23. Ohlsson $K$, Bjork $P$, Bergenfeldt $M$, Hageman R, Thompson RC. (1990) Interleukin-1 receptor antagonist reduces mortality from endotoxin shock. Nature 348: 550-552.

24. Bernhagen J, Calandra T, Mitchell RA, et al. (1993) MIF is a pituitary-derived cytokine that potentiates lethal endotoxaemia. Nature 365: 756-759.

25. Heinzel FP. (1990) The role of IFN- $\gamma$ in the pathology of experimental endotoxemia. $J$. Immunol. 145: 2920-2924.

26. Doherty GM, Lange JR, Langstein HN, Alexander HR, Buresh CM, Norton JA. (1992) Evidence for IFN- $\gamma$ as a mediator of the lethality of endotoxin and tumor necrosis factor- $\alpha$. J. Immunol. 149: 1666-1670. 